\title{
Correction to: Network-Based Integrated Analysis of Transcriptomic Studies in Dissecting Gene Signatures for LPS-Induced Acute Lung Injury
}

\author{
Fang Cao ${ }^{1}$, Chunyan Wang ${ }^{2}$, Danling Long ${ }^{3}$, Yujuan Deng ${ }^{4}$, Kaimin Mao ${ }^{5,7}$ and \\ Hua Zhong ${ }^{6}$
}

\section{Correction to: Inflamation \\ https://doi.org/10.1007/S10753-021-01518-8}

The original publication of this article contained mistakes. There should be an article note that will indicate that both authors' Fang Cao and Chunyan Wang contributed equally to the paper. Thus, the below statement should be captured as article note:

Fang Cao and Chunyan Wang contributed equally to this work.

The original article has been corrected.

Publisher's Note Springer Nature remains neutral with regard to jurisdictional claims in published maps and institutional affiliations.

The original article can be found online at https://doi.org/10. 1007/s10753-021-01518-8.

${ }^{1}$ Department of Cerebrovascular Disease, Affiliated Hospital of Zunyi Medical University, Huichuan District, 149 Dalian Road, Guizhou 563003 Zunyi, China

${ }^{2}$ Department of Gastroenterology, Sichuan Provincial Peoples Hospital, University of Electronic Science and Technology, Sichuan 610000, Chengdu, China

${ }^{3}$ Department of Stomatology, Taihe Hospital, Hubei University of Medicine, Hubei 442000, Shiyan, China

\footnotetext{
${ }^{4}$ School of Computer Science and Engineering, Shijiazhuang University, Shijiazhuang, Hebei, China

${ }^{5}$ Department of Critical Care Medicine, School of Medicine, Renji Hospital, Shanghai Jiaotong University, Shanghai 200127, China

${ }^{6}$ College of Life Sciences, Wuhan University, Wuhan 430072, Hubei, China

${ }^{7}$ To whom correspondence should be addressed at Department of Critical Care Medicine, School of Medicine, Renji Hospital, Shanghai Jiaotong University, Shanghai, 200127, China. Email: mkm444931158@126.com
} 